CAR-TR-553

October 1992

CS-TR-2662

\title{
Investigating Touchscreen Typing: The effect of keyboard size on typing speed
}

\author{
Andrew Sears \\ Doreen Revis \\ Janet Swatski \\ Rob Crittenden \\ Ben Shneiderman
Department of Computer Science \& Human-Computer Interaction Lab University of Maryland College Park, MD 20742

\begin{abstract}
Two studies investigated the effect keyboard size has on typing speed and error rates for touchscreen keyboards using the lift-off strategy. A cursor appeared when users touched the screen and a key was selected when they lifted their finger from the screen. Four keyboard sizes were investigated ranging from $24.6 \mathrm{~cm}$ to $6.8 \mathrm{~cm}$ wide. Results indicate that novices can type approximately 10 words per minute (WPM) on the smallest keyboard and 20 WPM on the largest. Experienced users improved to 21 WPM on the smallest keyboard and 32 WPM on the largest. These results indicate that, although slower, small touchscreen keyboards can be used for limited data entry when the presence of a regular keyboard is not practical. Applications include portable pocket-sized or palmtop computers, messaging systems, and personal information resources. Results also suggest the increased importance of experience on these smaller keyboards.

Research directions are suggested.
\end{abstract}




\section{Introduction}

Touchscreens are being used in a growing number of situations: information kiosks, banking machines, office directories, financial systems, and even by news analysts for the national news (Sears, Plaisant, \& Shneiderman, 1992). One of the motivations for this research is to continue exploring the possibility of using touchscreens in even more situations, such as palmtop and pocket-sized computers, portable message systems, and personal information resources.

Currently, the primary method for data entry is the standard QWERTY keyboard. This works well for computers that are stationary; however, as computers continue to become smaller and more portable, alternative methods for data entry must be investigated. Touchscreens may provide an interesting alternative. Touchscreens can be used with pocket-sized or palmtop computers, something that is difficult or impossible with a mouse. Touchscreens also allow the interface to be customized to fit the user and the task. For instance, users could specify the keyboard layout, language, and size that they wish to work with, something that is impossible with a traditional keyboard. By specifying a smaller touchscreen keyboard users can make additional screen space available for other uses which could be important on a portable computer with a small screen. In addition, the keyboard can be displayed only when data entry is required, allowing additional space to be used for displaying data.

Since there are many situations where the portable computer may be smaller than a traditional keyboard, alternative input devices must be investigated. The flexibility of touchscreen keyboards suggests that they may be appropriate for such situations. These studies investigated the effect keyboard size has on typing performance and user preference for touchscreen keyboards. Four keyboard sizes were investigated, ranging from 24.6 to $6.8 \mathrm{~cm}$ from the Q to $\mathrm{P}$ keys. Based on several previous studies that are discussed in the following section, we expected that typing would become slower as the keyboard became smaller (Sears, 1991; Sears \& Shneiderman, 1991). Time, errors, and preference ratings were collected to assess user performance and satisfaction. 


\section{Method}

\subsection{Subjects}

Twenty-four seniors and graduate students from the Computer Science Department of the University of Maryland volunteered to participate in the first experiment as novice subjects. All were familiar with the QWERTY keyboard, averaging 8.6 years of typing experience.

Four seniors and graduate students from the Computer Science Department of the University of Maryland participated in the second experiment as experienced users. All subjects had moderate experience using the touchscreen keyboards. Since these subjects had extensive knowledge of the purpose of the experiment subjective ratings were not gathered for this second experiment.

\subsection{Equipment}

2.2.1 Hardware: An NEC PowerMate 386/25 PC with a Sony Multi-scan HG monitor and MicroTouch touchscreen was used for both experiments. A special desk allowed the monitor to be mounted below the surface of the desk at various angles (Figure 1). The keyboard fit into a drawer which slid into the desk when not in use. The display area of the monitor measured $24.6 \times 18.3 \mathrm{~cm}$ and was used in VGA mode $(640 \times 480$ pixels). The MicroTouch touchscreen is a capacitive touchscreen that provides continuous information about the location of a touch on a $1024 \times 1024$ grid. It requires only a light touch to be activated and averages the location of all simultaneous touches, returning a centroid location. The touchscreen was cleaned once before the first subject began each experiment and was not cleaned again.

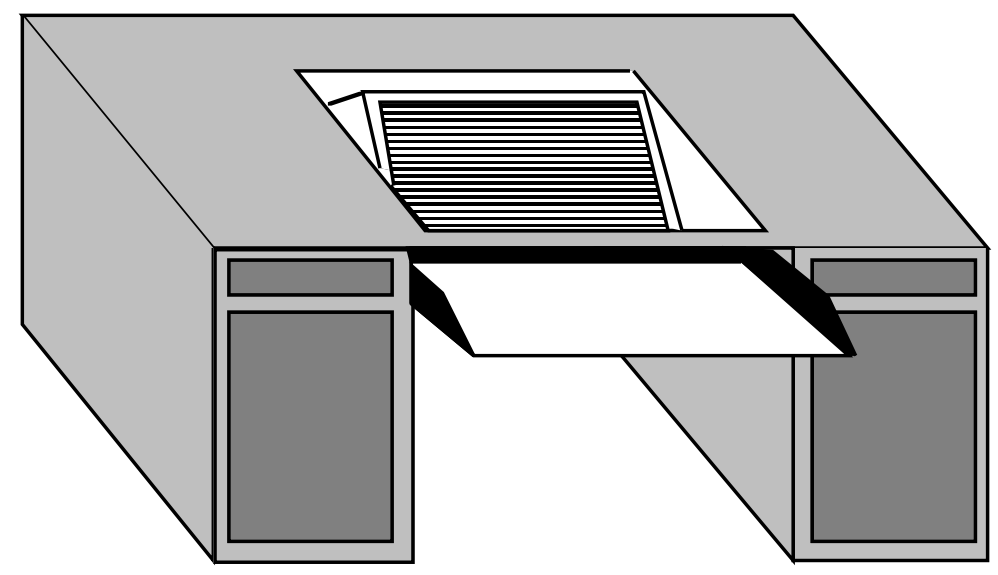

Figure 1 - Desk used in experiment 
2.2.2 Monitor Position: A recent study by Sears (1991) investigated touchscreen keyboards. This study suggested that the standard monitor position is sub-optimal, at least when using a touchscreen. The results indicated that mounting the touchscreen so the surface of the screen makes a 30 degree angle with the surface of the desk may result in reduced fatigue and increased user preference ratings. These results are also supported by work conducted by Ahlström and Lenman (1991). For this reason, the monitor used in these studies was mounted at 30 degrees.

2.2.3 Key Size and Selection Strategy: Sears (1991) also investigated the minimum size keys necessary to yield over $99 \%$ accuracy when using the land-on strategy. The results indicate that square keys must be approximately $2.27 \mathrm{~cm}$ per side to capture over $99 \%$ of a user's touches. These results are similar to those reported by Pfauth and Priest (1981), and by Hall, Cunningham, Roache, and Cox (1988) who recommended $2.2 \mathrm{~cm}$ and $2.6 \mathrm{~cm}$ per side respectively.

Using this information, we can conclude that reducing the size of keys below approximately $2 \mathrm{~cm}$ per side will result in unacceptable error rates if the land-on strategy were used. Since small keyboards are of particular interest, we resorted to an alternate selection strategy, lift-off. When using the lift-off selection strategy, a cursor appears when a user touches the screen. The user can then drag the cursor to the desired location and a selection is made where they lift their finger from the screen (Potter, Weldon, \& Shneiderman, 1988). The lift-off strategy has been shown to be fast and accurate for targets approximately $0.2 \mathrm{~cm}$ per side (Sears \& Shneiderman, 1991).

\subsection{Design and Procedure}

Four keyboard sizes were used, with alphabetic keys measuring 2.27, 1.14, 0.76, and 0.57 $\mathrm{cm}$ per side. The largest size, $2.27 \mathrm{~cm}$, was chosen to result in the largest keyboard that could be displayed on the available monitor. The remaining three sizes are $50 \%, 33 \%$, and $25 \%$ of the largest key size. The space bar, backspace and done keys were all proportional in size. Number and punctuation keys were not included on the current keyboards to allow comparisons with the previous study by Sears (1991). Future studies will investigate complete keyboards (Plaisant \& Sears, 1992). The keyboards measured 24.6, 13.2, 9.0, and $6.8 \mathrm{~cm}$ from the $\mathrm{Q}$ to $\mathrm{P}$ keys. Keys on traditional keyboards are approximately $1.35 \mathrm{~cm}$ per side and measure approximately $19.0 \mathrm{~cm}$ from the Q to $\mathrm{P}$ key. Keyboards appeared as shown in Figure 2 and will be referred to as Large, Medium, Small, or Extra Small (L, M, S, XS). The string that users were to type appeared $3.2 \mathrm{~cm}$ above the keyboard, and the string the users actually typed appeared $1.9 \mathrm{~cm}$ below the string to be 
typed (Figure 2). The touchscreen used the lift-off selection strategy, making selection of the smallest keys possible. The cursor was placed directly below the user's finger when using the three larger keyboards, and slightly above the user's finger when using the Extra Small keyboard. This difference was necessary to allow users to see the key they were selecting when using the Extra Small keyboard. Both visual and audible feedback were provided to users. When a key was touched, it highlighted indicating that the key would be selected if the user lifted their finger. When subjects did select a key, the key returned to normal colors and a clicking sound was made.

THE QUICK BROWN FOX JUMPED OVER THE LAZY DOG

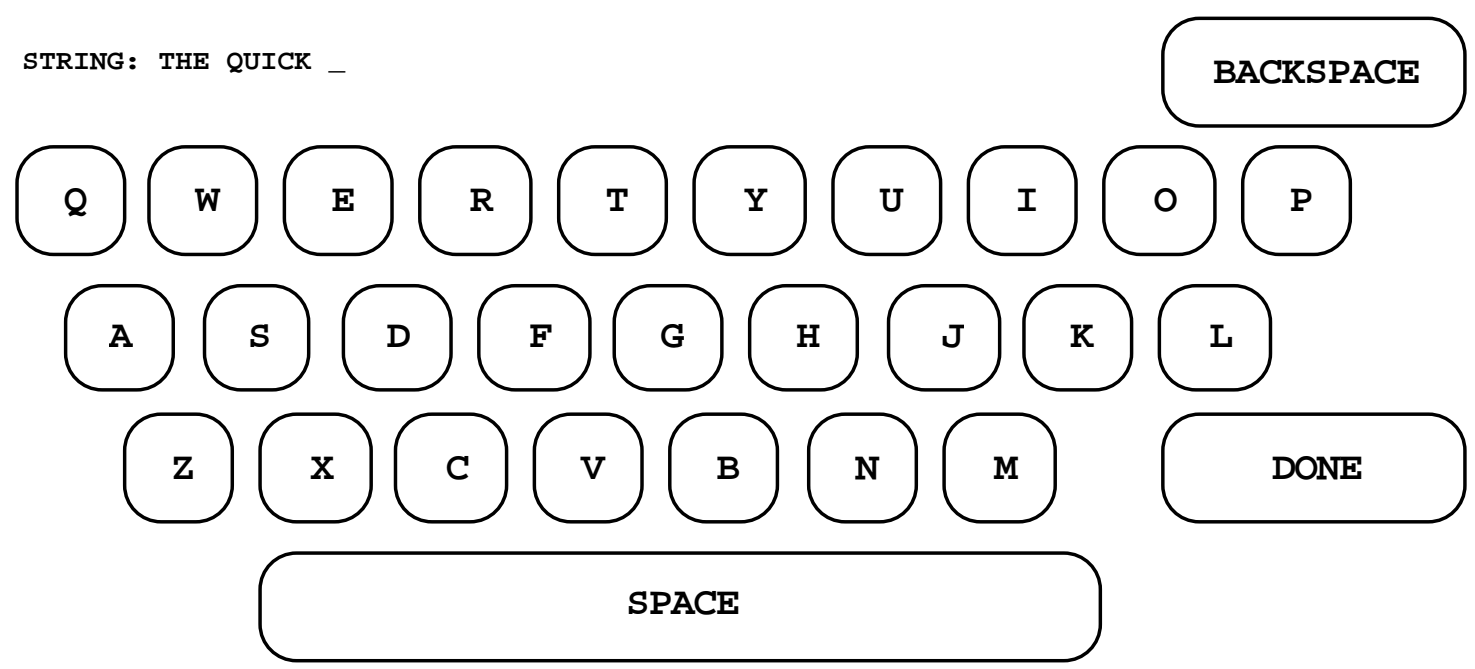

Figure 2 - Keyboard layout and string positions

Keyboard size and stings entered were within subject variables. Every subject entered one practice string and three additional strings for which data were collected with each of the keyboard sizes (Table 1 contains the three strings for which data were collected). Keyboard size was randomized to prevent any biases. The time from when the first character of each string was typed until the done key was touched was automatically recorded. In addition, the number of corrected and uncorrected errors were also recorded. A sequence of backspaces was considered one corrected error. Any error in the final string was considered an uncorrected error. The time, corrected errors, and uncorrected errors for each set of three strings using each keyboard size were recorded for each subject. In addition, subjects in the novice user experiment answered several questions after completing the experiment.

\section{MONDAY}




\section{FIRST WE MUST START}

\section{THE QUICK BROWN FOX JUMPED OVER THE LAZY DOG}

Table 1 - Three strings for which data were collected

The first experiment used novice subjects, providing estimates of typing rates for first time users with minimal experience with touchscreen keyboards. The subjects in second experiment had a minimum of one-half hour of experience using the touchscreen keyboards and are referred to as experienced. The experienced users participated several times each, and the fastest time for each keyboard size was recorded. These results are intended to represent optimal performance for users with moderate experience.

\section{Results}

\subsection{Typing Rates}

Mean time to complete all three strings with each keyboard size appears, with standard deviations, in Table 2. Equivalent Words Per Minute rates (assuming 5 characters per word) appear in Table 3 and Figure 3. An ANOVA with repeated measures for keyboard size showed a significant main effect for WPM rates for both experiments, $F(3,69)=70.4(p<.001)$ for novices and $\mathrm{F}(3,9)=25.5$ ( $\mathrm{p}<.001)$ for experienced users. Tukey's post hoc HSD test showed that performance was significantly different on all keyboard sizes for novices (Tukey's critical range $=1.96, \mathrm{p}<.05)$. Tukey's post hoc HSD test also showed that the Large keyboard was faster than the three smaller keyboards and the Medium keyboard is faster than the Extra Small keyboard for the experienced users (Tukey's critical range $=4.15, \mathrm{p}<.05$ ).

The total time until completion was transformed using a logarithmic function and additional ANOVAs were performed for both experiments. There were no differences between these results and those of the first ANOVA of the novice users $(F(3,69)=67.6, p<.001$, Tukey's critical range $=0.06, \mathrm{p}<.05)$. There was one additional significant difference for the experienced users; the Small keyboard was faster than the Extra Small keyboard $(F(3,9)=33.8, \mathrm{p}<.001$, Tukey's critical range $=0.07, \mathrm{p}<.05)$.

$\begin{array}{lllll} & \text { Extra Small } & \text { Small } & \text { Medium } & \text { Large } \\ \text { Novices } & 93.0(24.2) & 67.2(17.6) & 55.2(15.0) & 43.7(7.7) \\ \text { Experienced } & 41.1(2.9) & 35.0(3.1) & 32.3(1.4) & 26.9(3.0)\end{array}$

Table 2 - Total time (in seconds) to type three strings for novices and experienced users (standard deviation in parentheses) 


$\begin{array}{lllll} & \text { Extra Small } & \text { Small } & \text { Medium } & \text { Large } \\ \text { Novices } & 9.9(2.4) & 13.7(3.5) & 16.5(3.6) & 20.3(3.3) \\ \text { Experienced } & 21.1(1.5) & 24.9(2.1) & 26.8(1.2) & 32.5(3.7)\end{array}$

Table 3 - WPM for novices and experienced users by keyboard size (standard deviations in parentheses).

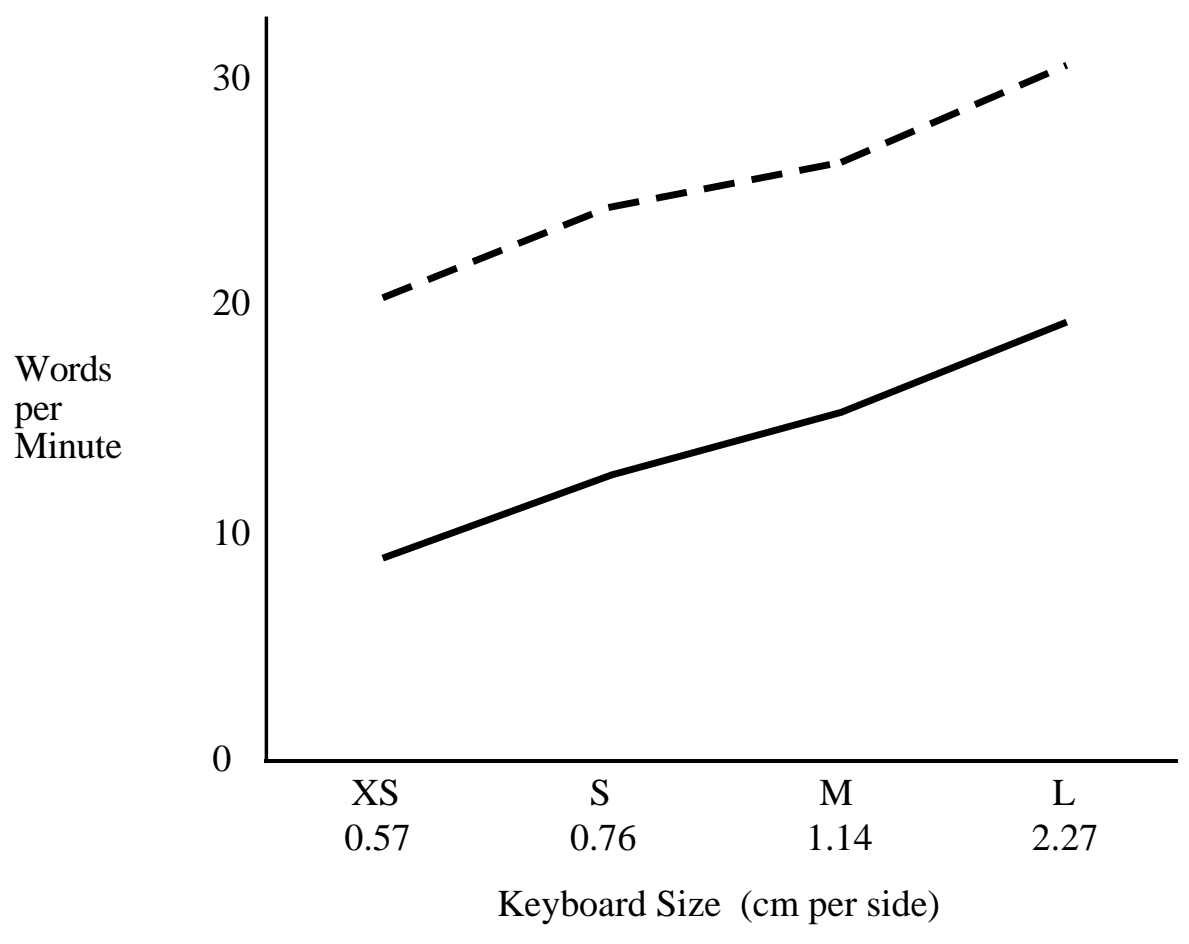

Figure 3 - Words per minute by keyboard size

- - - Experienced - Novice

\subsection{Error Rates}

Errors were divided into two categories: corrected and uncorrected. The mean for corrected and uncorrected errors for each keyboard size appear, with standard deviations, in Tables 4 and 5. Errors from all three strings were combined for each keyboard size. An ANOVA with repeated measures for keyboard size was performed on corrected and uncorrected errors for the novice user experiment. A significant main effect was found for keyboard size on corrected errors, $\mathrm{F}(3,69)=4.5$ ( $\mathrm{p}<.01)$. Tukey's post hoc HSD showed that the Large keyboard resulted in significantly fewer corrected errors than the Extra Small keyboard (Tukey's critical range=1.72, $\mathrm{p}<.05)$. No significant differences were found for uncorrected errors for novice users. 
An ANOVA with repeated measures for keyboard size showed no significant effects for corrected errors for the experienced user experiment. Due to the lack of uncorrected errors for experienced users, statistical comparisons were not performed.

$\begin{array}{lllll} & \text { Extra Small } & \text { Small } & \text { Medium } & \text { Large } \\ \text { Novices } & 4.5(4.5) & 3.3(3.3) & 3.4(2.8) & 2.2(1.7) \\ \text { Experienced } & 1.3(1.0) & 1.5(1.0) & 1.3(1.0) & 0.3(0.5)\end{array}$

Table 4 - Mean total corrected errors for novices and experienced users (standard deviation in parentheses)

$\begin{array}{lllll} & \text { Extra Small } & \text { Small } & \text { Medium } & \text { Large } \\ \text { Novices } & 0.1(0.3) & 0.4(0.7) & 0.4(0.6) & 0.3(0.9) \\ \text { Experienced } & 0.0(0.0) & 0.3(0.5) & 0.0(0.0) & 0.0(0.0)\end{array}$

Table 5 - Mean uncorrected errors for novices and experienced users (standard deviation in parentheses)

\subsection{Subjective Satisfaction - Novices}

Results of three subjective questions appear in Table 6. Overall, novice subjects thought the Large keyboard was the easiest to type on, the Medium was the nicest in appearance, and the Large and Medium were rated the highest for overall preference. Mean comfort ratings appear in Table 7 ( 1 = least comfortable to use, $9=$ most comfortable to use). The Large keyboard was rated the most comfortable to use, and Extra Small least comfortable.

$\begin{array}{lllll} & \text { Extra Small } & \text { Small } & \text { Medium } & \text { Large } \\ \text { Ease of typing } & 0 & 1 & 5 & 18 \\ \text { Appearance } & 0 & 1 & 15 & 8 \\ \text { Overall } & 0 & 2 & 11 & 11\end{array}$

Table 6 - Number of subjects that thought each keyboard size was the best in each category
Comfort
Extra Small
Small
Medium
6.0
Large
3.5
7.8

Table 7 - Mean comfort rating for each keyboard size

\subsection{Discussion}

As expected, the smaller the keyboard, the longer it took to type the strings. It is clear that 
moderate experience and practice on the tasks can dramatically improve typing rates, WPM rates increased by $11.2,11.2,10.3$, and 12.2 for the XS, S, M, and L keyboard sizes respectively. However, if this is presented as the percent increase in WPM for each keyboard size the importance of experience is more clear. Users improved by $113,82,62$, and $60 \%$ for the XS, S, $\mathrm{M}$, and L keyboards respectively. This indicates that limited experience results in a $60 \%$ increase for the Large keyboard. However, the same experience resulted in almost twice as much improvement, $113 \%$, for the Extra Small keyboard. This clearly demonstrates the benefit of even limited experience with touchscreen keyboards.

These results compare favorably with those reported by Sears (1991), where subjects used a keyboard the same size as the Large keyboard in the current studies. Subjects used the keyboard three to five times, which was more than the novices but less than the experienced users in the current studies. Subjects typed an average of 25.4 WPM after this limited experience which falls between the values for novices and experienced users in the current studies.

Subjective ratings clearly favored the larger keyboards. Subjects felt that the Large keyboard was the easiest to type on and the most comfortable to use. As the keyboards got smaller, satisfaction ratings decreased.

The experimenters observed the subjects during this experiment to determine the methods subjects used when typing on the various keyboards. After typing only one practice string, subjects quickly selected a typing strategy they felt was the most successful. The majority of subjects used one or two fingers from each hand on the three smaller keyboards, while approximately half of the subjects used most of their fingers to type on the Large keyboard.

Capacitive touchscreens have been reported to be the slowest technology to respond to touches (Carroll, 1989). As previously reported, it appears that the slow response time of the capacitive touchscreen may have limited typing rates (Sears, 1991). Currently, users must delay slightly between consecutive touches. If a faster technology or one that allowed simultaneous touches were used, faster typing rates may be possible. A description of how Fitts' Law may apply to touchscreens appears in Sears and Shneiderman (1991).

\section{Conclusions}

These studies provide an initial measure of the effect key size has on performance with touchscreen keyboards. Although typing rates were slower than those possible with traditional keyboards, users with moderate experience were able to type as many as 32 WPM using the Large keyboard. These same users could type 21 WPM on even the Extra Small keyboard. In addition, the number of uncorrected errors was low for the novices and was reduced to zero for three of the 
four keyboards for the experienced users. Although these typing rates are slower than traditional keyboards, we believe that these results demonstrate the potential for touchscreen keyboards in many special situations including: small portable computers (pocket-sized or palmtop), or when keyboards are not durable enough, data entry is limited, or users may want to select the type of keyboard to use (QWERTY, Dvorak, Alphabetic, French, Swedish, etc.).

The results indicate that performance and preference both increase as the keyboard becomes larger. Performance and preference using the Medium and Large keyboards were similar, indicating that either keyboard could be used effectively. However, even the Extra Small keyboard could be used if screen space is severely limited, such as on a pocket calendar. To take full advantage of the flexibility of the touchscreen, it may be desirable to allow users to specify the exact size of the keyboard at run time. This could be accomplished by simply resizing the keyboard in the same way a window is resized.

\subsection{Future Research}

There are many opportunities for research in this area. First, various size mechanical keyboards could be compared to equivalent size touchscreen keyboards. Although the typing rates were not very high for the smallest touchscreen keyboard in these studies, one must wonder how fast users could type on a mechanical keyboard of the same size. This is interesting when considering applications with limited physical space such as the Casio BOSS.

As mentioned previously, the touchscreen technology used in these studies is relatively slow responding to a users touch. Repeating these studies or conducting similar studies using a faster technology may result in improved typing rates.

Additional research must be conducted to determine the optimal touchscreen monitor position. Sears (1991) conducted a study which indicates that subjects prefer to use a touchscreen when it is mounted at approximately 30 degrees from horizontal. However, several subjects felt that the desk used in these studies forced them to lean too far forward. This may be due to the size and position of the monitor. It is possible that using a flat monitor with a touchscreen may be more comfortable.

\section{Acknowledgements}

We would like to thank Catherine Plaisant and John Smelcer for their comments, and the subjects for their participation and patience. We would also like to thank NCR Corporation for partial support of this research. 


\section{References}

Ahlström, B., Lenman, S., and Marmolin, T. (1991). Overcoming touchscreen user fatigue by workplace design (Technical Report). Stockholm, Sweden: Royal Institute of Technology, Department of Computer Science.

Carroll Touch. (1989). Touch Handbook. Round Rock, TX: Carroll Touch Inc.

Hall, A., Cunningham, J., Roache, R., and Cox, J. (1988). Factors affecting performance using touch-entry systems: tactal recognition fields and system accuracy. Journal of Applied Psychology, 73, 4, 711-720.

Pfauth, M., and Priest, J. (1981). Person-computer interface using touch screen devices. Proceedings of the 25th Annual meeting of the Human Factors Society (pp. 500-504), Santa Monica, CA: Human Factors Society.

Plaisant, C., and Sears, A. (1992, October). Touchscreen interfaces for flexible alphanumeric data entry. To appear in: Proceedings of the 36th Annual meeting of the Human Factors Society, Santa Monica, CA: Human Factors Society.

Potter, R., Weldon, L., Shneiderman, B. (1988, May). Improving the Accuracy of Touch Screens: An Experimental Evaluation of Three Strategies. Proceedings of the conference on Human Factors in Computing Systems (CHI'88) (pp. 27-32), New York: ACM.

Sears, A. (1991). Improving Touchscreen Keyboards: Design issues and a comparison with other devices. Interacting with Computers, 3, 3, 252-269.

Sears, A., Shneiderman, B. (1991). High-precision touchscreens: Design strategies and comparisons with a mouse. International Journal of Man-Machine Studies, 34, 4, 593-613.

Sears, A., Plaisant, C., \& Shneiderman, B. (1992). A new era for high-precision touchscreens. To appear in: R. Hartson \& D. Hix (Eds.), Advances in Human-Computer Interaction (Volume 3), Norwood, NJ: Ablex Publishing Company. 
